\title{
Flujos de plasma hidrodinámicos y magnetohidrodinámicos. Parte III
}

\section{Hydrodynamic and magnetohydrodynamic plasma flows. Part III}

\section{Néstor Rotstein}

Estudio de energías por fusión de plasmas magnetizados, Facultad Regional Buenos Aires, Universidad Tecnológica Nacional - Argentina

nrotstein@frba.utn.edu.ar

\section{Ignacio Bustelo Cancela}

Estudio de energías por fusión de plasmas magnetizados, Facultad Regional Buenos Aires, Universidad Tecnológica Nacional - Medrano 951 (CP1179) - Buenos Aires - Argentina ignaciobustelo@gmail.com

\section{Resumen}

En este artículo hallamos la expresión general de la topología que el campo magnético adopta para poder limitar las corrientes que se generan a lo largo de sus líneas en plasmas con resistividad no nula. A los efectos de comparar con modelos de plasmas ideales asumimos componente azimutal de la fuerza de Lorentz nula. Mostramos que la intensidad del campo magnético no depende del coeficiente de difusión, como sí lo hacen el campo eléctrico inducido y el flujo másico asociado. El desacople de las isosuperficies de flujo de masa y las superficies de flujo magnético constante hace que el problema matemático no sea ya de variables separables, pero mostramos las condiciones que deben cumplir la densidad de masa (que deja de ser esféricamente simétrica) y el campo de velocidades.

Palabras clave: plasmas - magnetohidrodinámico - topología - resistividad

\begin{abstract}
In this article we find the general expression of the topology that the magnetic field adopts in order to limit the currents that are generated along its lines in plasmas with non-zero resistivity. In order to compare with ideal plasma models we assume null Lorentz force azimuthal component. We show that the magnetic field strength does not depend on the magnetic diffusivity coefficient, as do the induced electric field and the associated mass flux. The decoupling of mass flux isosurfaces and constant magnetic flux surfaces means that the mathematical problem is no longer one of separable variables, but we show the conditions
\end{abstract}


that the mass density (which is no longer spherically symmetric) and the velocity field must satisfy.

Keywords: plasmas - magnetohydrodynamic - topology - resistivity

\section{Introducción}

Una de las características distintivas de la interacción entre un plasma idealmente conductor en movimiento y un campo magnético externo, es que las líneas del campo magnético, arrastradas por el fluido, conservan indefinidamente su identidad, sin importar qué tan retorcidas puedan llegar a estar siguiendo el movimiento del fluido. En estas condiciones se dice que el fluido arrastra a la líneas, o que éstas se hallan "congeladas" al plasma y lo contienen. Bajo la hipótesis de conductividad ideal, entonces, el escenario es el de un fluido magnetizado que evoluciona embebido en un campo magnético, pero sin cambios en la topología del campo magnético.

El congelamiento de las líneas de campo magnético al campo de velocidades constituye la base del confinamiento magnético. En términos poco rigurosos, hablamos de confinamiento magnético cuando se limita la evolución espacial de un plasma mediante la interacción con un campo magnético externo. Si las líneas de campo están cerradas, el plasma habrá de evolucionar idealmente siguiendo la misma trayectoria cerrada. Al cabo es esa la idea primigenia de un generador de energía por fusión. Habitualmente se trata de dispositivos con simetría axial (axisimetría) como por ejemplo tokamak, stellarator, spheromak, o RFP (que son las siglas de reversed field pinch). En estos dispositivos el objetivo está puesto en lograr que las partículas de plasma colisionen por tiempos comparativamente grandes en volúmenes comparativamente reducidos, y esto sin interactuar con las paredes del recinto que contiene al campo (y al plasma confinado).

Sin embargo, la condición de congelamiento es muy fuerte y no siempre es posible asumirla, fundamentalmente porque podrían existir configuraciones de mínima energía que no se alcanzarían en la hipótesis de conductividad infinita. De hecho, un equilibrio dinámicamente estable desde el punto de vista de la MHD ideal puede llegar a ser inestable si en el tratamiento se incluyen los efectos de la resistividad.

Lo que ocurre es que las fluctuaciones propias de las variables dinámicas pueden generar diversas inestabilidades, algunas de ellas capaces de romper el confinamiento. Ahora bien, el confinamiento magnético no es exclusivo de las máquinas de fusión, y de hecho los plasmas confinados magnéticamente son habituales a escala astrofísica. La diferencia esencial es que mientras que en los objetos astrofísicos los eventos disruptivos originados por efecto de inestabilidades resistivas constituyen una característica (en todo caso capaz de explicar diversos procesos de calentamiento, por ejemplo) en los generadores por fusión son, por definición, inadmisibles.

En las máquinas de fusión el confinamiento se logra en cámaras de geometría toroidal mediante superficies magnéticas cerradas. El campo magnético toroidal es generado por medio de bobinas externas, y un solenoide ubicado en el eje del toroide genera una variación temporal en el flujo magnético poloidal, induciendo de este modo una fuerza electromotriz toroidal que produce la corriente del plasma. Esta corriente, que genera la componente poloidal del campo magnético de confinamiento, contribuye a la formación de superficies anidadas, en las que idealmente, en estado de equilibrio MHD, el gradiente de 
presión compensa a la fuerza de Lorentz.

Sin embargo, la resistividad no nula del plasma (que es equivalente a decir difusividad no nula) provoca necesariamente la reconfiguración del campo magnético de equilibrio. $Y$ en plasmas confinados magnéticamente esta reconfiguración puede ser crucial. La difusividad provoca que las líneas de corriente que idealmente serían paralelas al campo magnético tiendan a deformarse, juntándose en algunas regiones y separándose en otras, lo que provoca la aparición de líneas de campo cerradas que antes no existían. Este "desgarramiento" de la topología original da origen a un tipo de inestabilidad disruptiva que se conoce precisamente como tearing mode.

Estos modos constituyen una importante fuente de inestabilidad de la topología, que lleva a un proceso de relajación que puede resultar directamente en el transporte de calor y partículas y, en general, a procesos disruptivos del confinamiento en los que las superficies de flujo se rompen en "islas magnéticas" y enfrían al plasma.

A escala astrofísica también ocurren fenómenos disruptivos, básicamente en regiones las que existen gradientes de corriente muy intensos (como pueden ser los filamentos magnéticos o en las hojas de corriente en la corona solar) regiones en las que, sea por alguna inestabilidad o por el movimiento propio del plasma, las líneas de campo magnético de regiones topológicamente diferentes se ponen en contacto y reconectan de un modo diferente, las estructuras magnéticas cerradas ya no son posibles, los arcos se abren y liberan la energía contenida, convirtiendo energía magnética en energía cinética y térmica del plasma.

Pero en tanto que en el Sol el plasma se libera y simplemente abandona la atmósfera, en un tokamak se enfría y la fusión se interrumpe, de ahí la necesidad de controlar que el plasma no escape del confinamiento y que la energía no se pierda.

La primera consecuencia que se desprende de estas observaciones es que la topología del campo magnético debe adaptarse a la existencia de resistividad porque en la hipótesis de resistividad no nula el flujo magnético a través de una superficie fluida no puede permanecer constante, que es lo mismo que decir que la componente de velocidad del fluido perpendicular a las líneas de campo no puede ser la misma que la de las líneas. Desde el punto de vista de la ley de Faraday este comportamiento es de esperar, porque si un conjunto de cargas libres (típicamente un plasma) atraviesa líneas de campo magnético habrá de inducir un campo eléctrico a lo largo de las mismas líneas, de modo tal que se generarán corrientes eléctricas que sólo van a verse limitadas por la resistividad del fluido.

De esta forma, parece tener sentido preguntarse cuál es la topología de equilibrio estacionario de un plasma resistivo embebido en un campo magnético, porque en un plasma que no sea idealmente conductor las líneas de campo magnético no tienen por qué coincidir en todo punto con las líneas del campo de velocidades (el campo magnético difunde a través de las líneas de flujo).

En cierta medida porque en las partes I y II de este trabajo hemos tratado problemas de flujos de plasma en geometría esférica, en parte porque el tratamiento no sólo es un poco más sencillo que en geometría toroidal sino que además disponemos de evidencia observacional, en este artículo habremos de continuar en la hipótesis de una configuración axisimétrica en geometría esférica con fuerzas gravitatorias (más emparentada a objetos estelares), dejando para el próximo artículo el tratamiento axisimétrico en geometría cilíndrico anular (más emparentada a jets o a tokamaks) porque el objetivo está enfocado a estudiar los efectos de la resistividad del plasma. Por lo pronto, habremos de demostrar que la topología estacionaria del campo magnético está directamente relacionada con la existencia de las 
corrientes paralelas a las líneas de campo pero que, a diferencia del campo eléctrico inducido, no depende del valor de la resistividad o del coeficiente de difusión.

Otra de las ventajas de tratar el problema en escalas astrofísicas es que a esa escala no es esperable un campo magnético que difunda en tiempos observables. Si bien los tiempos de difusión dependen de las características dinámicas y estructurales de cada sistema (por ejemplo, el tiempo de difusión del campo magnético interior terrestre es del orden de $10^{5}$ años, en tanto que el tiempo de difusión del campo magnético solar es del orden de $10^{10}$ años. Respecto a las escalas de tiempo y espacio involucradas véase, por ejemplo, Biskamp, 1996, o Tenerani et al, 2016) elegimos la escala astrofísica porque nuestro interés no está puesto en la difusión en sí misma sino en el modo en que el campo debe ajustarse a la condición impuesta de corrientes eléctricas paralelas a sus líneas. Lo que procuramos en este artículo es hallar esa configuración de equilibrio, y comparar nuestros resultados con los campos magnéticos que se fijan idealmente a priori a partir de un criterio de plausibilidad, hipótesis que en el caso resistivo carecerían de sentido.

Para ello, en el capítulo 2 plantearemos las ecuaciones generales del problema, que resolveremos en el apartado 3. En la sección 4 mostraremos que el campo de velocidades necesariamente queda desacoplado del campo magnético, es decir, las isosuperficies de flujo másico ya no coinciden con las isosuperficies de flujo magnético. Y que a diferencia del campo magnético, el flujo de masa depende del valor de la resistividad. Las conclusiones y consecuencias de este fenómeno las discutimos en la sección 5.

\section{Tratamiento general}

Las ecuaciones magnetohidrodinámicas que gobiernan la evolución estacionaria de un plasma no ideal, interactuando con campos magnéticos externos, en presencia de un campo gravitatorio, se escriben como

$$
\begin{aligned}
& \nabla \cdot \vec{B}=0 \\
& \nabla \cdot \rho \vec{v}=0 \\
& \nabla \times\left(\vec{v} \times \vec{B}-\frac{\vec{j}}{\sigma}\right)=0 \\
& \rho(\vec{v} \cdot \nabla) \vec{v}=-\nabla \mathcal{P}+\frac{1}{\mu_{0}}(\nabla \times \vec{B}) \times \vec{B}-\rho g \hat{e}_{r}
\end{aligned}
$$

donde, como es habitual, $\rho$ representa la densidad volumétrica de masa, $\vec{v}$ el campo de velocidades, $\vec{B}$ el campo de inducción magnética, $g$ la aceleración gravitatoria, $\mathcal{P}=k_{B} \rho T / \mu m$ la presión del fluido ( $\mu$ es el peso molecular medio, $\mathrm{k}_{\mathrm{B}}$ la constante de Boltzmann) y $\sigma$ la conductividad eléctrica.

Notemos que independientemente del valor de la conductividad, la ecuación (1) de divergencia nula del campo magnético, y la ecuación de continuidad (2) pueden escribirse como 


$$
\begin{aligned}
& \vec{B}=\frac{1}{r \operatorname{sen} \theta} \nabla A(r, \theta) \times \hat{e}_{\varnothing}+B_{\varnothing} \hat{e}_{\varnothing} \\
& \rho \vec{v}=\frac{1}{r \operatorname{sen} \theta} \nabla \psi(r, \theta) \times \hat{e}_{\varnothing}+\rho V_{\varnothing} \hat{e}_{\varnothing}
\end{aligned}
$$

donde hemos introducido las funciones (escalares) $A(r, \theta)$ y $\psi(r, \theta)$, función de flujo magnético y función de flujo másico, respectivamente. Estas funciones gobiernan la topología de las superficies de flujo constante, magnético y másico en cada caso. Las superficies en las que $A=$ constante y $\psi=$ constante contienen las proyecciones de las líneas de campo magnético y de flujo sobre el plano meridional. Más aún, las isosuperficies magnéticas son superficies electrostáticas equipotenciales. Para un plasma idealmente conductor $(\sigma \rightarrow \infty)$ la ecuación (3) afirma que, además, el campo de velocidades evoluciona también paralelo al campo magnético, esto es, si la función potencial electrostático es constante el campo poloidal de velocidades debe coincidir en cada punto con el campo magnético poloidal (Chandrasekhar, 1956; Tsinganos,1982), esto es

$$
v_{P}=\varepsilon B_{P}
$$

donde $\varepsilon$ es una función escalar que depende de la posición en el plano poloidal. La conductividad finita impide la coincidencia de las isosuperficies de flujo, y en tal caso ya no puede invocarse que el momento angular total sea constante sobre una línea meridional, esto es, la condición

$$
r \operatorname{sen} \theta v_{\phi}-\frac{r \operatorname{sen} \theta B_{\phi}}{\rho \varepsilon}=\ell
$$

donde 1 es una constante sobre una línea de campo, ya no es válida, de manera tal que ya no podremos escribir las componentes toroidales del campo en términos de las funciones $A(r, \theta)$ y $\psi(r, \theta)$. En términos prácticos, en nuestra formulación hemos perdido dos ecuaciones, de manera tal que en lugar de estipular una relación particular entre las dos funciones de flujo deberemos estipular (siempre en base a un criterio de plausibilidad) al menos una de las dos componentes toroidales del campo, ya sea el magnético o el de velocidades. Por otro lado, las componentes meridionales del campo magnético pueden obtenerse a partir de la expresión (5) en la forma

$$
\begin{aligned}
& B_{r}=\frac{\partial_{\theta} A}{r^{2} \operatorname{sen} \theta} \\
& B_{\theta}=-\frac{\partial_{r} A}{r \operatorname{sen} \theta}
\end{aligned}
$$

que definen formalmente las componentes poloidales del campo magnético una vez establecida la función de flujo magnético. La estructura angular de las ecuaciones (9) y (10) adelantan que esta función debe poseer cierta simetría poloidal en el sentido de que cada 
hemisferio sea similar al otro, de manera tal que debe ser proporcional a alguna potencia $\operatorname{sen}^{2 n} \theta$ del ángulo polar. La forma más general posible será entonces

$$
A(r, \theta)=\sum_{n \geq 1} \omega_{2 n}(r) \operatorname{sen}^{2 n} \theta
$$

A pesar de ser completa en cuanto al desarrollo angular la expresión (11) presupone un desarrollo muy extenso. Como nuestra prioridad es ilustrar los efectos que la resistividad tiene sobre la topología del campo magnético, trataremos sólo el primer término de la jerarquía, es decir, propondremos la forma

$$
A(r, \theta)=\omega_{2}(r) \operatorname{sen}^{2} \theta
$$

porque además de contener la esencia del problema mantiene el tratamiento matemático en un nivel razonable. Dejaremos para el final la discusión sobre términos superiores. Obsérvese un detalle de importancia, en las partes I y II de este trabajo impusimos la forma de la función $\omega_{2}(r)$, en este caso es una incógnita del problema a resolver.

Las componentes de la densidad de corriente

$$
\vec{j}=\frac{1}{\mu_{0}} \nabla \times \vec{B}=\frac{1}{\mu_{0}} \nabla \times\left[\frac{1}{r \operatorname{sen} \theta} \nabla A(r, \theta) \times \hat{e}_{\varnothing}+B_{\varnothing} \hat{e}_{\varnothing}\right]
$$

se escriben en consecuencia como

$$
\begin{aligned}
& j_{r}=\frac{1}{\mu_{0} r \operatorname{sen} \theta} \partial_{\theta}\left(B_{\phi} \operatorname{sen} \theta\right) \\
& j_{\theta}=-\frac{1}{\mu_{0} r} \partial_{r}\left(r B_{\phi}\right) \\
& j_{\phi}=\frac{1}{\mu_{0} r}\left[\partial_{r}\left(r B_{\theta}\right)-\partial_{\theta} B_{r}\right]
\end{aligned}
$$

Como hemos hecho en los trabajos anteriores, introduciremos una distancia adimensional en términos del radio del objeto, esto es, $x=r / R_{*}$, de manera tal que las derivadas respecto de la coordenada radial las redefiniremos como

$$
\partial_{r} \equiv \frac{\partial}{\partial r}=\frac{1}{R_{\star}} \partial_{x}
$$

Definamos ahora la cantidad 


$$
\beta=x B_{\phi} \operatorname{sen} \theta
$$

y con el objetivo de no sobrecargar la notación convengamos en primar las derivadas respecto a la distancia adimensional $x$. De esta manera podemos escribir las ecuaciones (14)-(16) en la forma

$$
\begin{aligned}
& j_{r}=\frac{1}{\mu_{0} R_{\star} x^{2} \operatorname{sen} \theta} \partial_{\theta} \beta \\
& j_{\theta}=-\frac{1}{\mu_{0} R_{\star} x \operatorname{sen} \theta} \beta^{\prime} \\
& j_{\phi}=\frac{1}{\mu_{0} R_{\star}^{3} x^{3}}\left(2 \omega_{2}-x^{2} \omega_{2}^{\prime \prime}\right) \operatorname{sen} \theta=\frac{1}{\mu_{0} R_{\star} x^{3}}\left(2 W_{2}-x^{2} W_{2}^{\prime \prime}\right)
\end{aligned}
$$

donde hemos definido $W_{2}=\omega_{2} / R_{\star}^{2}$ para trabajar con variables de similar orden de magnitud. Regresemos ahora a la ecuación (3), y escribámosla en términos de la función escalar potencial electrostático $Y(x, \theta)$ en la forma

$$
\vec{v} \times \vec{B}-\frac{\vec{j}}{\sigma}=\nabla \mathrm{Y}(x, \theta)
$$

o, equivalentemente

$$
\frac{\vec{J}}{\sigma} \cdot \vec{B}+\vec{B} \cdot \nabla \mathrm{Y}=0
$$

A partir de la expresión (23) parece evidente que en el caso de un plasma idealmente conductor $(\sigma \rightarrow \infty)$ el potencial eléctrico debe ser fijado como una constante a través de cada superficie de flujo magnético. En el caso que estamos trabajando, en cambio, debemos postular las formas de las funciones $\beta(x, \theta)$ y $Y(x, \theta)$, esto es, en base a algún criterio debemos estipular la manera en que evolucionan espacialmente la componente toroidal del campo magnético y el campo eléctrico. Un breve análisis de la geometría con la que estamos trabajando sugiere tomar

$$
\nabla \mathrm{Y}(x, \theta)=F(x) \cos ^{t} \theta \quad t \in \mathbb{Z}>1
$$

para mantener el problema en variables separadas. A su vez, y por razones similares, parece razonable definir

$$
\beta(x, \theta)=\beta_{r}(x) \operatorname{sen}^{p} \theta \quad p \in \mathbb{Z}>1
$$


Los parámetros $t$ y $p$ definen el número de ecuaciones independientes que es posible obtener del problema si tomamos una función potencial magnético como la de la expresión (12), aunque en principio bastará con tomar sólo una de ellas como función trigonométrica de alto orden. Dado que el papel de la función $\beta(x ; \theta)$ no se limita a definir la componente toroidal del campo magnético $\mathrm{B}_{\varphi}$ (entre las ecuaciones (18) y (25)) sino que también denota la forma del campo de velocidad rotacional (a través de la componente azimutal de la ecuación (4)) y la forma de las líneas de corriente poloidales (a través de las ecuaciones (19) y (20)), parece razonable fijar la función de potencial eléctrico y dejar las potencias variables para la función $\beta(x ; \theta)$. En consecuencia, a partir de ahora, adoptaremos $z=1$.

Teniendo en cuenta estas observaciones, la ecuación (23), después de un poco de álgebra, se convierte en

$$
\begin{aligned}
& \eta\left[\frac{\beta_{r}}{x^{4}} W_{2}\left(2 p \operatorname{sen}^{p-2} \theta+(2-p) \operatorname{sen}^{p} \theta\right)+\frac{\beta_{r}^{\prime}}{x^{2}} W_{2}^{\prime} \operatorname{sen}^{p} \theta-\frac{\beta_{r}}{x^{2}} W_{2}^{\prime \prime} \operatorname{sen}^{p} \theta\right]+ \\
& +\left[2 \frac{F^{\prime}}{x^{2}} W_{2}\left(1-\operatorname{sen}^{2} \theta\right)+\frac{F}{x^{2}} W_{2}^{\prime} \operatorname{sen}^{2} \theta\right]=0
\end{aligned}
$$

donde hemos introducido el coeficiente de difusividad magnético $\eta=1 / \mu_{0} \sigma$.

\section{La topología del campo magnético}

La ecuación (25) nos permite una primera mirada del rol que juega la resistividad (o en la manera en que lo escribimos, la difusividad) en la topología del campo magnético. Por simple inspección, puede verse que la ecuación (25) queda correctamente dimensionada para $p=4$, o para $p=2$ y una relación definida entre dos variables cualesquiera.

Demostraremos a continuación que en el primer caso no existe solución física posible. En efecto, para $p=4$, la ecuación (26) se descompone Fourier como

$$
\begin{aligned}
& \left\{\frac{7}{4} \frac{W_{2} \beta_{r}}{x^{4}}+\frac{3}{8} \frac{W_{2}^{\prime} \beta_{r}^{\prime}}{x^{2}}-\frac{3}{8} \frac{W_{2}^{\prime \prime} \beta_{r}}{x^{2}}+\frac{W_{2} F^{\prime}}{\eta x^{2}}+\frac{W_{2}^{\prime} F}{2 \eta x^{2}}\right\}+ \\
& +\left\{-\frac{W_{2} \beta_{r}}{x^{4}}-\frac{1}{2} \frac{W_{2}^{\prime} \beta_{r}^{\prime}}{x^{2}}+\frac{1}{2} \frac{W_{2}^{\prime \prime} \beta_{r}}{x^{2}}+\frac{W_{2} F^{\prime}}{\eta x^{2}}-\frac{W_{2}^{\prime} F}{2 \eta x^{2}}\right\} \cos 2 \theta+ \\
& +\left\{-\frac{3}{4} \frac{W_{2} \beta_{r}}{x^{4}}+\frac{1}{8} \frac{W_{2}^{\prime} \beta_{r}^{\prime}}{x^{2}}-\frac{1}{8} \frac{W_{2}^{\prime \prime} \beta_{r}}{x^{2}}\right\} \cos 4 \theta=0
\end{aligned}
$$

que en virtud del tercer término puede escribirse como

$$
4 \frac{W_{2} \beta_{r}}{x^{4}}+\frac{W_{2} F^{\prime}}{\eta x^{2}}+\frac{W_{2}^{\prime} F}{2 \eta x^{2}}=0
$$




$$
\begin{aligned}
& -4 \frac{W_{2} \beta_{r}}{x^{4}}+\frac{W_{2} F^{\prime}}{\eta x^{2}}-\frac{W_{2}^{\prime} F}{2 \eta x^{2}}=0 \\
& -3 \frac{W_{2} \beta_{r}}{x^{4}}+\frac{1}{2}\left(\frac{W_{2}^{\prime} \beta_{r}^{\prime}}{x^{2}}-\frac{W_{2}^{\prime \prime} \beta_{r}}{x^{2}}\right)=0
\end{aligned}
$$

Las ecuaciones (28) y (29) muestran que si alguna solución existe es con $F=$ constante, y que en tal caso resulta

$$
\beta_{r}=-\frac{F}{8 \eta} \frac{W_{2}^{\prime}}{W_{2}}
$$

que, reemplazada en la expresión (30) conduce a la ecuación en $W_{2}$

$$
W_{2}^{\prime}\left[\frac{3}{x^{2}}-\frac{1}{x} \frac{W_{2}^{\prime}}{W_{2}}+\frac{1}{2}\left(\frac{W_{2}^{\prime}}{W_{2}}\right)^{2}\right]=0
$$

que, como puede verse fácilmente por su propia estructura matemática, no tiene solución real (o la tiene, pero es la solución sin significado físico $W_{2}^{\prime}=0$ ). Luego, debemos explorar las soluciones con $p=2$.

En tal caso, la expresión (26) deriva en la forma

$$
\begin{aligned}
& \eta\left[4 \frac{W_{2} \beta_{r}}{x^{4}} \cos ^{2} \theta+\frac{W_{2}^{\prime} \beta_{r}^{\prime}}{x^{2}} \operatorname{sen}^{2} \theta-\frac{\beta_{r}}{x^{2}}\left(\frac{2 W_{2}}{x^{2}}-W_{2}^{\prime \prime}\right) \operatorname{sen}^{2} \theta\right]+ \\
& +\frac{2 W_{2} F^{\prime}}{x^{2}} \cos ^{2} \theta+\frac{W_{2}^{\prime} F}{x^{2}} \operatorname{sen}^{2} \theta=0
\end{aligned}
$$

que de inmediato se desarrolla Fourier como

$$
\begin{aligned}
& \left\{\left[4 \eta \frac{W_{2} \beta_{r}}{x^{4}}+\frac{2 W_{2} F^{\prime}}{x^{2}}\right]+\left[\eta\left(\frac{W_{2}^{\prime} \beta_{r}^{\prime}}{x^{2}} \operatorname{sen}^{2} \theta-\frac{\beta_{r}}{x^{2}}\left(\frac{2 W_{2}}{x^{2}}-W_{2}^{\prime \prime}\right)\right)+\frac{W_{2}^{\prime} F}{x^{2}}\right]\right\}+ \\
& +\left\{\left[4 \eta \frac{W_{2} \beta_{r}}{x^{4}}+\frac{2 W_{2} F^{\prime}}{x^{2}}\right]-\left[\eta\left(\frac{W_{2}^{\prime} \beta_{r}^{\prime}}{x^{2}} \operatorname{sen}^{2} \theta-\frac{\beta_{r}}{x^{2}}\left(\frac{2 W_{2}}{x^{2}}-W_{2}^{\prime \prime}\right)\right)+\frac{W_{2}^{\prime} F}{x^{2}}\right]\right\} \cos 2 \theta=0
\end{aligned}
$$

Es casi evidente que la única manera de satisfacer la ecuación (34) es que cada término entre paréntesis sea nulo por separado, de lo contrario los términos de la serie de Fourier resultante no podrían ser nulos. Entonces, la ecuación (34) se reduce a las dos expresiones siguientes 
Asumiendo $W_{2}^{\prime} \neq 0$ la expresión (36) puede escribirse en la forma sucinta

$$
\eta\left(\beta_{r}^{\prime}+\beta_{r} f\left(W_{2}\right)\right)+F=0
$$

donde hemos definido

$$
f\left(W_{2}\right)=\frac{\frac{2 W_{2}}{x^{2}}-W_{2}^{\prime \prime}}{W_{2}^{\prime}}
$$

Derivando una vez la ecuación (37) y por medio de la expresión (35), tras un sencillo pero engorroso álgebra se puede establecer la relación completa entre $\beta_{r}(x)$ y $f\left(W_{2}\right)$ en la forma

$$
\beta_{r}^{\prime \prime}+\beta_{r}^{\prime} f\left(W_{2}\right)+\beta_{r}\left[f\left(W_{2}+\frac{2}{x}\right)\right]^{\prime}=0
$$

de modo que la función $W_{2}(x)$ puede hallarse una vez que se determina la forma funcional de $\beta_{r}(x)$ sobre la base de alguna hipótesis adicional. Para poder comparar con los modelos de plasmas idealmente conductores, supondremos que el flujo evoluciona sin componente toroidal de la fuerza de Lorentz.

En este punto posiblemente sea necesario decir que usualmente en los modelos de flujo poloidal se toma $B_{\varphi}=0$, lo que inmediatamente conduce a que las componentes poloidales de la corriente sean nulas y, en consecuencia, la componente azimutal de la fuerza de Lorentz sea idénticamente nula. No es nuestro caso, nosotros estamos adoptando una particular relación entre las componentes poloidales del campo magnético y de la corriente, precisamente la que hace que una componente de la fuerza de Lorentz sea nula. Y no podríamos suponer $B_{\varphi}=0$ porque en tal caso las corrientes poloidales serían nulas (véanse las expresiones (19) y (20)) que son precisamente las que modifican la topología del campo magnético debido a la resistividad no nula.

La condición de componente toroidal de la fuerza de Lorentz nula se escribe como

$$
(\vec{j} \times \vec{B})_{\phi}=j_{r} B_{\theta}-j_{\theta} B_{r}=\frac{B_{\theta}}{\mu_{0} R_{\star} x^{2} \operatorname{sen} \theta} \frac{\partial \beta}{\partial \theta}+\frac{B_{r}}{\mu_{0} R_{\star} x \operatorname{sen} \theta} \frac{\partial \beta}{\partial x}=0
$$

que puede escribirse de una manera más compacta en la forma

$$
(\vec{j} \times \vec{B})_{\phi}=\frac{\partial \beta}{\partial x} \frac{\partial A}{\partial \theta}-\frac{\partial \beta}{\partial \theta} \frac{\partial A}{\partial x}=0
$$

de donde puede verse de manera casi inmediata que la hipótesis de componente azimutal nula de la fuerza de Lorentz es equivalente a plantear que la función $\beta(x, \theta)$ es función de la función potencial flujo magnético $A(x, \theta)$, esto es, $\beta(x, \theta)=f[A(x, \theta)]$ independientemente de la forma que adopte el potencial de flujo magnético. En nuestro caso, esta condición se reduce 
a la forma

$$
\beta(x, \theta)=\frac{A(x, \theta)}{R_{\star}^{2}}=W_{2}(x) \operatorname{sen}^{2} \theta
$$

De esta manera, la ecuación (39) se escribe como

$$
W_{2}^{\prime \prime}+W_{2}^{\prime} f\left(W_{2}\right)+W_{2} f^{\prime}\left(W_{2}\right)=\frac{2 W_{2}}{x^{2}}
$$

o, lo que es equivalente

$$
f\left(W_{2}\right)-\frac{\frac{2 W_{2}}{x^{2}}-W_{2}^{\prime \prime}}{W_{2}^{\prime}}=\frac{W_{2}}{W_{2}^{\prime}} f^{\prime}\left(W_{2}\right)
$$

A partir de la definición (38) es inmediato que el lado derecho de la última ecuación debe anularse, por lo que la función $f\left(W_{2}\right)$ debe ser una constante, digamos $m$, y, en este marco particular, la ecuación para $W_{2}(x)$ resulta

$$
W_{2}^{\prime \prime}+m W_{2}^{\prime}-\frac{2 W_{2}}{x^{2}}=0
$$

cuya solución, como puede comprobarse por reemplazo directo, resulta en la forma

$$
W_{2}(x)=c_{1} e^{-m x}\left(1+\frac{2}{m x}\right)+c_{2}\left(\frac{m}{2}-\frac{1}{x}\right)
$$

donde $c_{1}$ y $c_{2}$ son constantes en principio arbitrarias. Puede verse que la solución es válida para todo valor de $m \neq 0$, en cuyo caso la solución

$$
W_{2}(x)=\frac{c_{3}}{x}+c_{4} x^{2}
$$

es casi inmediata, con $c_{3}$ y $c_{4}$ constates también en principio arbitrarias.

La figura 1 muestra las líneas de campo para $m=0,5, m=1$ y $m=1,5$. El panel $d$ representa la superposición de un campo puramente radial y uno dipolar, que resulta para $m=-1, c_{1}=0, c_{2}=-1$. La torsión de las líneas de campo es evidente, especialmente en el panel $b$, un efecto que sólo tiene que ver con la resistividad y que proviene del término exponencial de la ecuación (46). Nótese que, en términos generales el valor del parámetro $m$ determina la latitud por debajo de la cual el material puede expandirse hacia regiones del disco ecuatorial, y eventualmente regresar a la superficie del objeto central. De hecho, cuanto menor sea $\mathrm{m}$, mayor será esta latitud, a partir de la cual el plasma se aleja grandes distancias en forma expansiva. 
Mencionemos de nuevo que la solución que presentamos se basa en la hipótesis de que la componente toroidal de la fuerza de Lorentz es nula. Lo que no podemos imponer es la condición $B_{\varphi}=0$ porque sería equivalente a formular un modelo ideal con resistividad nula. Pero debe quedar claro que, aunque no es para nada limitativa, no es más que una hipótesis de trabajo.

Por ejemplo, podríamos trabajar con una componente toroidal de campo magnético que sea decreciente con la distancia. Esta es también una hipótesis realista y plausible, de forma tal que podríamos escribir

$$
\beta_{r}=\beta_{0} x^{q}+\kappa \quad(q<1)
$$

donde $\kappa$ es una constante positiva que hemos introducido para que eventualmente $B_{\varphi}$ tenga un término decreciente lento. Los valores permitidos de q tienen que ver con el hecho de que, a diferencia de $B_{\varphi}, \beta(x)$ no es necesariamente una función decreciente. Definiendo $b=\kappa / \beta_{0}$ la ecuación (39) se transforma en

$$
-\frac{q(1-q)}{x^{2-q}}+\frac{q}{x^{1-q}} f\left(W_{2}\right)+\left(x^{q}+b\right)\left[f\left(W_{2}\right)+\frac{2}{x}\right]^{\prime}=0
$$

cuyas soluciones en términos de q y b son casi inmediatas para $f\left(W_{2}\right)$ en las formas

$$
\begin{aligned}
& f\left(W_{2}\right)=\frac{1}{b+x^{q}}\left[\lambda+\frac{c_{q}}{(2 q-1) x^{1-2 q}}-\frac{b\left(c_{q}+2\right)}{(1-q) x^{1-q}}-\frac{2 b^{2}}{x}\right] \quad q \neq 1 / 2 \\
& f\left(W_{2}\right)=\frac{1}{b+x^{1 / 2}}\left[\lambda-\frac{2 b^{2}}{x}-\frac{13}{2} \frac{b}{x^{1 / 2}}+\frac{9}{4} \ln x\right] \quad q=1 / 2
\end{aligned}
$$

donde $\lambda$ es una constante arbitraria y $c_{q}$ una función del parámetro $q$ definida como

$$
c_{q}=2+q(1-q)
$$

Es casi evidente que existe ahora un conjunto de soluciones que dependen del particular del parámetro $q$. A modo de ejemplo, para $q=-1, b=1$, resulta

$$
W_{2}(x)=\frac{c_{5}}{x}\left[e^{-\frac{\lambda}{2} x^{2}}+3 \sum_{n \geq 1} \frac{(-1)^{n}}{(2 n+1) ! !} \lambda^{n-1} x^{2 n+1}\right]+c_{6} x^{2}\left[\sum_{n \geq 0} \frac{3(-1)^{n}}{(2 n+3) ! !} \lambda^{n} x^{2 n}\right]
$$

donde $c_{5}$ y $c_{6}$ es otro par de constantes arbitrarias. En términos generales la integración numérica del conjunto de ecuaciones para $W_{2}(x)$ (a partir de la relación (38)), dependiendo de los valores de las constantes involucradas, resulta en topologías similares a las de la figura 1. Hay sin embargo dos diferencias destacables. La pimera es que las soluciones dependen ahora de cuatro constantes arbitrarias, y no tres, como en el caso de componente 
azimutal nula de la fuerza de Lorentz. Y la segunda, y más importante, es la forma funcional de la velocidad de rotación. En la sección siguiente lo justificaremos.

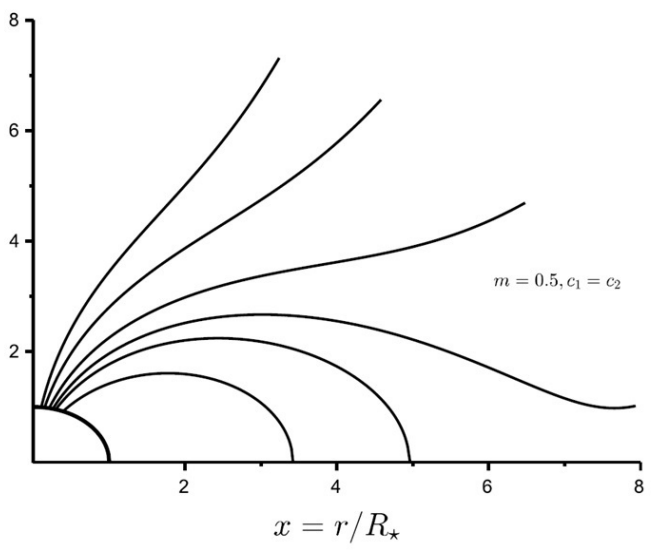

(a)

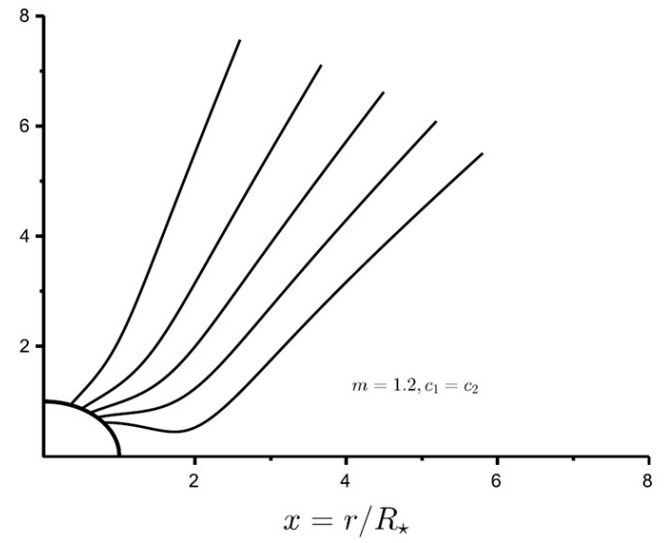

(c)

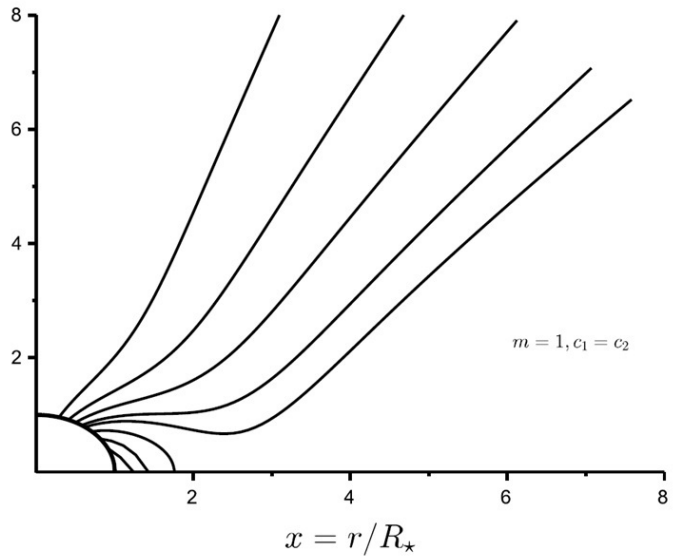

(b)

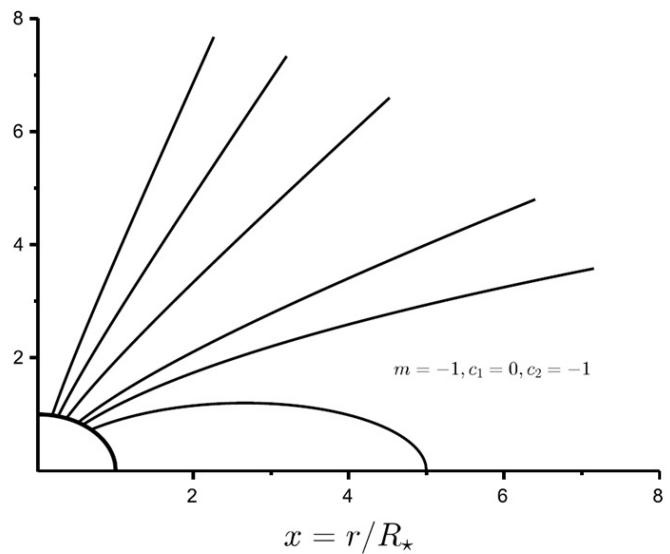

(d)

Figura 1: líneas de campo magnético para diferentes valores del parámetro de curvatura m. En el panel (c) puede verse que la topología deja de ser cerrada a partir de $m \approx 1,2$. El panel (d) muestra las líneas de un campo magnético puramente dipolar superpuesto a uno puramente radial ( $\left.m=-1, c_{1}=0, c_{2}=-1\right)$ con resistividad nula. La diferencia fundamental entre el tratamiento ideal y el resistivo puede verse entre los paneles (a) y (d): con resistividad no nula las líneas se curvan hacia el polo después de formar una región de líneas cerradas.

\section{El flujo asociado}

Escribamos el conjunto completo de ecuaciones para el flujo de masa. Para la función magnética (12) las componentes radial y polar del campo de velocidad pueden escribirse a partir de la ley de conservación de la masa, ecuación (2), utilizando la expresión (6), esto es

$$
\nabla(\rho \vec{v})=0 \Rightarrow\left\{\begin{array}{c}
v_{r}=\frac{\partial \psi / \partial \theta}{\rho R_{\star}^{2} x^{2} \sin \theta} \\
v_{\theta}=-\frac{\partial \psi / \partial x}{\rho R_{\star}^{2} x \sin \theta}
\end{array}\right.
$$


Por otro lado, la componente azimutal de la ecuación (3) se escribe como

$$
v_{r} B_{\theta}-v_{\theta} B_{r}=\frac{1}{\sigma} j_{\phi}
$$

que en términos de las expresiones (9), (10) y (16) puede escribirse en la forma

$$
\frac{\partial \psi / \partial x}{\rho x^{2} \sin \theta} \frac{2 W_{2}}{W_{2}^{\prime}} \operatorname{cotg} \theta-\frac{\partial \psi / \partial \theta}{\rho x^{2} \sin \theta}=\alpha \frac{\frac{2 W_{2}}{x^{2}}-W_{2}^{\prime \prime}}{W_{2}^{\prime}} \alpha f\left(W_{2}\right)
$$

de manera que es inmediata la forma de la densidad $\rho(x, \theta)$

$$
\rho(x, \theta)=\frac{1}{\alpha f\left(W_{2}\right) x^{2} \sin \theta}\left[\frac{\partial \psi}{\partial x} \frac{2 W_{2}}{W_{2}^{\prime}} \operatorname{cotg} \theta-\frac{\partial \psi}{\partial \theta}\right]
$$

donde hemos definido la constante $\alpha=R, \mu_{0} \sigma$. Un par de comentarios respecto de la ecuación (57) se hacen necesarios. En primer lugar, de haber tomado $\alpha=0$ (o equivalentemente $\sigma \rightarrow \infty)$ la única solución posible sería que se anule el corchete, es decir, que sea $\psi(x, \theta)=h(A(x, \theta))$. De esta manera es casi evidente que lo que cambia radicalmente el problema es la constante $\alpha$.

Específicamente, podríamos reescribir la condición (57) en la forma

$$
[\psi, A]=W_{2}^{\prime} \operatorname{sen}^{2} \theta\left\{\frac{\partial \psi}{\partial x} \frac{2 W_{2}}{W_{2}^{\prime}} \operatorname{cotg} \theta-\frac{\partial \psi}{\partial \theta}\right\}=\frac{1}{\sigma} j_{\phi} \rho x^{2} \operatorname{sen}^{2} \theta
$$

y de nuevo, el operador sólo será nulo si el plasma es idealmente conductor $\sigma \rightarrow \infty$ ) o $\operatorname{si} j_{\varphi}=0$, condición que se cumple si, y sólo si, el parámetro m de la ecuación (45) es nulo. Luego, en estas condiciones es imposible que la función flujo másico $\psi(x, \theta)$ pueda escribirse en términos de la función potencial de campo magnético $A(x, \theta)$. Y de hecho hemos fijado la función de potencial electrostático a través de la denición (24). Sobre la base de estos vínculos, proponemos una función flujo másico de la forma

$$
\psi(x, \theta)=\psi_{0}\left(a \cos \theta+u(x) \operatorname{sen}^{2} \theta\right)
$$

donde $\psi_{0}$ y a son dos constantes (la segunda es adimensional), y $u(x)$ una función adimensional y en principio libre, porque de esta manera el problema conserva identidad. En estas condiciones, la ecuación de la densidad de masa (57) resulta

$$
\rho(x, \theta)=\frac{\psi_{0}}{\alpha f\left(W_{2}\right) x^{2}}[a+2 g(x) \cos \theta]
$$


donde por comodidad hemos definido la función

$$
g(x)=u^{\prime} \frac{W_{2}}{W_{2}^{\prime}}-u
$$

Dos características resultan inmediatas. En primer lugar, la dependencia angular de la función densidad anticipa que el signo de la constante a debe ser el mismo que el de la función $f\left(W_{2}\right)$. Por otro lado, es casi evidente que no pueden ser simultáneamente $a=0 \mathrm{y}$ $u(x)=W_{2} / W_{0}$ ( $W_{0}$ es una constante). En estas condiciones, las componentes polares de la velocidad resultan

$$
\begin{aligned}
& v_{r}(x, \theta)=v_{0} f\left(W_{2}\right) \frac{-a+2 u(x) \cos \theta}{a+2 g(x) \cos \theta} \\
& v_{\theta}(x, \theta)=-v_{0} f\left(W_{2}\right) \frac{x u^{\prime}(x) \cos \theta}{a+2 g(x) \cos \theta}
\end{aligned}
$$

donde hemos definido $v_{0}=\alpha / R_{\star}^{2}=1 / \mu_{0} \sigma R_{\star}$. Se observa que en la región ecuatorial y en su vecindad puede existir una componente radial entrante del flujo, que en principio podría representar material que fluye para formar algún tipo de disco o caer hacia la superficie del objeto central. La existencia de dicha región ecuatorial está

relacionada con los signos de $v_{r}$ y de $v_{\theta}$, que es lo mismo que decir que depende del signo de $f\left(W_{2}\right)$ y de $u(x)$. Por ejemplo, este tipo de plasma entrante existirá para el campo magnético que hemos encontrado en la expresión (46). Y la extensión en el plano polar de esta región depende naturalmente de $u(x)$ y del valor del parámetro a. Dicho en otras palabras, para una distancia relativa $x_{p}$, la velocidad radial desaparece (y cambia de signo) en un ángulo dado por $\cos \theta=a / 2 u\left(x_{p}\right)$.

Escribamos formalmente ahora la ecuación (4). Teniendo en cuenta la expresión (6), resulta

$$
v_{r} \frac{\partial V_{\phi}}{\partial x}+\frac{v_{\theta}}{x} \frac{\partial V_{\phi}}{\partial \theta}+\frac{V_{\phi} v_{r}}{x}+\frac{V_{\phi} v_{\theta}}{x} \operatorname{cotg} \theta=\frac{1}{R_{\star} \rho \mu_{0} x^{3} \operatorname{sen}^{2} \theta}\left[\frac{\partial \beta}{\partial x} \frac{\partial A}{\partial \theta}-\frac{\partial \beta}{\partial \theta} \frac{\partial A}{\partial x}\right]
$$

Obsérvese ahora que con la hipótesis $[\vec{j} \times \vec{B}]_{\phi}=0$ (el corchete del miembro derecho de la ecuación (64)) la solución de la componente azimutal de la es inmediata. En efecto, empleando las formas (62) y (63) y pensando la componente toroidal en la forma genérica $V_{\varphi}=v_{\varphi}(x) G(\theta)$, escribiremos la última expresión como

$$
-a\left(v_{\phi}^{\prime}+\frac{v_{\phi}}{x}\right) G(\theta)+2 u\left(v_{\phi}^{\prime}+\frac{v_{\phi}}{x}\right) G(\theta) \cos \theta-u^{\prime} v_{\phi}\left[\frac{\partial G(\theta)}{\partial \theta} \operatorname{sen} \theta-G(\theta) \cos \theta\right]=0
$$


Ahora bien, sin importar a forma exacta de la función $\mathrm{G}(\theta)$, el primer sumando impone la condición

$$
v_{\phi}^{\prime}+\frac{v_{\phi}}{x}=0
$$

Por otro lado, no parece demasiado especulativo esperar que la velocidad de rotación tenga un máximo en el ecuador, de manera tal que parece razonable asumir una forma angular $G(\theta)=\operatorname{sen} \theta$ (una forma funcional que anula idénticamnete el corchete de la última expresión) en cuyo caso obtenemos

$$
V_{\phi}(x, \theta)=\frac{v_{0}}{x} \operatorname{sen} \theta
$$

donde $v_{\varphi 0}$ se refiere a la velocidad toroidal del flujo en $x=1, \theta=\pi / 2$. Obsérvese que esta forma (por lo demás plausible) sólo pudo obtenerse suponiendo que la componente azimutal de la fuerza de Lorentz es nula. En cualquier otro caso deberíamos haber definido previamente el valor de la constante a y la forma funcional de $u(x)$, con lo cual podríamos haber integrado previamente las funciones (62) y (63), un formalismo que no es sencillo porque no se trata sólo de criterios de plausibilidad, ya que aun cuando optáramos por esta vía restaría acoplar esas soluciones a las formas de la presión del plasma, una tarea que está fuera del alcance de este trabajo.

\section{Conclusiones}

En este trabajo hemos considerado la evolución de un plasma con resistividad no nula en geometría esférica. En primer lugar debería observarse que en los modelos con resistividad nula el campo magnético debe indicarse a priori, de una manera más o menos general. Si se toma $B_{\varphi}=0$ resulta $F_{L O R \varphi}=0$. En cambio, en este modelo es imposible tomar $B_{\varphi}=0$ porque en tal caso el modelo carece de sentido.

En principio podría parecer extraño o llamativo que el modelo no dependa de parámetro alguno que, tendiendo a cero, permita reducir este tratamiento al de plasma ideal. Pero precisamente esto se debe a que un caso no se reduce al otro. Si se considera resistividad nula, al menos en este escenario, es imposible hallar la topología del campo magnético porque perdemos la ecuación que lo vincula al campo de velocidades y, en consecuencia, debe ser definido a priori.

Pero es importante señalar que las líneas de campo magnético no se corresponden con las líneas de campo de velocidades, porque la hipótesis de resistividad no nula separa automáticamente las isosuperficies de flujo magnético de las de flujo másico.

Nuestro tratamiento no procura sostener que el campo magnético difunde a través del campo de flujo, de hecho en este contexto es un efecto menor. Lo que pretendemos resaltar es que la topología del campo es muy diferente a la que habitualmente se asume, y sólo en casos particulares puede reducirse a la de plasma ideal.

La integración de las ecuaciones dinámicas constituye ahora un verdadero desafío, por un lado porque las ecuaciones ya no son de variables separadas, y por el otro porque una elección arbitraria de la función $u(x)$, si bien podría parecer plausible, podría llevar a soluciones sin significado en las formas de la presión del flujo. 
Hemos demostrado que sólo en el caso de componente toroidal nula de la fuerza de Lorentz puede hallarse la forma funcional de la velocidad de rotación. No es esta un restricción limitativa, que la componente azimutal de la fuerza de Lorentz sea nula dice que cada elemento de plasma evoluciona siempre en un mismo plano. Obsérvese además que esto no significa que no haya componentes toroidales del campo magnético y del campo de velocidades, porque de hecho la que es nula es la aceleración azimutal, no la velocidad. De hecho cada elemento de fluido recorre cada paralelo con la misma velocidad toroidal.

Si bien el tratamiento ha sido planteado en coordenadas esféricas, es de destacar que aparecen ya aquí algunos elementos que se repiten en cualquier geometría. Tal vez el más importante sea el desacople de las superficies de flujo constante, lo que hace que en cualquier tipo de geometría las ecuaciones dinámicas no sean ya de variables separadas. El tratamiento en coordenadas cilíndrico toroidales (capaces de refrescar la imagen de un tokamak) requiere además de consideraciones adicionales, pero sin embargo también pueden hallarse algunas soluciones analíticas considerando que la fuerza de Lorentz tiene componente toroidal nula. Tengamos en cuenta que al desaparecer el término gravitatorio, en un plasma de fusión ideal la situación de equilibrio se presenta de manera sencilla en la forma $\nabla p=\vec{j} \times \vec{B}$. Esto significa que el gradiente de presión del plasma es perpendicular tanto a las corrientes como al campo externo, de manera tal que en un equilibrio MHD la presión y los contornos de flujo magnético coinciden, es decir, las líneas de campo magnético se apoyan sobre las superficies de presión constante. Por otro lado, en el caso ideal el campo eléctrico paralelo a las líneas de campo magnético es nulo, lo que lleva necesariamente a que las superficies de flujo másico constante coinciden con las de flujo magnético, a menos de una rotación rígida (porque $\vec{j} \cdot \nabla p=0$ implica que no existen componentes de $\vec{j}$ perpendiculares a las superficies de presión constante).

Sin embargo, al abandonar la hipótesis de conductividad infinita, las isosuperficies se separan y es ese fenómeno el que provoca el desacople de las ecuaciones, un tema que excede los objetivos de este artículo y que dejaremos para el siguiente. 


\section{Referencias}

Biskamp, D (1996): Astrophysics and Space Science, 242, 165. DOI 10.1007/BF00645113

Chandrasekhar, S (1956): PNAS, 42, 1. DOI 10.1073/pnas.42.1.1

Tenerani, A; Velli, M; Pucci, F; Landi, S; Rapazzo, A (2016): Journal of Plasma Physics, 82, article id. 535820501. DOI 10.10017/S002237781600088X

Tsinganos, K (1982): Astophys. J, 252, 775. DOI 10.1086/159600 